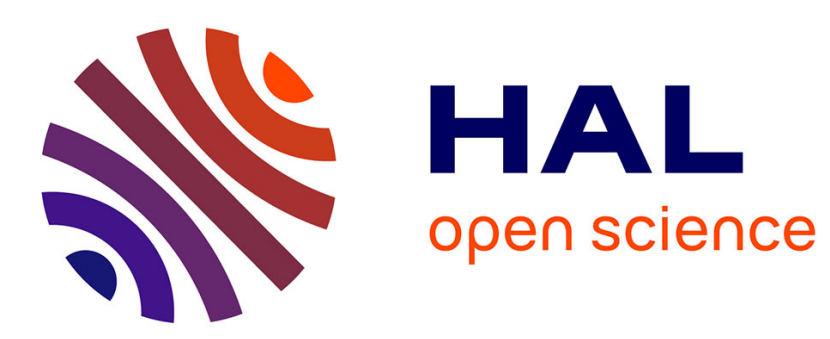

\title{
DIFFUSION ORTHOGONALE DES ONDES ULTRA-SONORES PAR LES HÉTÉROGÉNÉITÉS SUB-SURFACIQUES DE LA MER
}

\author{
C. Renard
}

\section{> To cite this version:}

C. Renard. DIFFUSION ORTHOGONALE DES ONDES ULTRA-SONORES PAR LES HÉTÉROGÉNÉITÉS SUB-SURFACIQUES DE LA MER. Journal de Physique IV Proceedings, 1992, 02 (C1), pp.C1-961-C1-964. 10.1051/jp4:19921210 • jpa-00251177

\section{HAL Id: jpa-00251177 https://hal.science/jpa-00251177}

Submitted on 1 Jan 1992

HAL is a multi-disciplinary open access archive for the deposit and dissemination of scientific research documents, whether they are published or not. The documents may come from teaching and research institutions in France or abroad, or from public or private research centers.
L'archive ouverte pluridisciplinaire HAL, est destinée au dépôt et à la diffusion de documents scientifiques de niveau recherche, publiés ou non, émanant des établissements d'enseignement et de recherche français ou étrangers, des laboratoires publics ou privés. 


\title{
DIFFUSION ORTHOGONALE DES ONDES ULTRA-SONORES PAR LES HETEROGENEITES SUB- SURFACIQUES DE LA MER
}

\author{
C. RENARD \\ Laboratoire d'Electronique Appliquée aux Capteurs, I.U.T. de Brest, F-29287 Brest cedex, France
}

\begin{abstract}
RESUME : Un système flottant bi-statique émet une onde U.S. continue à $150 \mathrm{kHz}$ dont le faisceau étroit coupe à angle droit a $50 \mathrm{~cm}$ sous la surface de la mer, le fin faisceau de réception. L'amplitude fluctuante de l'onde reçue après diffusion est analysée, modélisée et discutée. La turbulence convective isotrope semble être la cause de ces fluctuations.

ABSTRACT : A buoyant bi-static device transmits a continuous 150 kcs U.S. wave whose the narrow beam crosses with a right angle a narrow receiving beam at $50 \mathrm{~cm}$ under the sea surface. The fluctuating amplitude of the received scattered wave is processes, modelized and discussed. Convective isotropic turbulence seems to be the cause of these fluctuations.
\end{abstract}

\section{1 - INTRODUCTION}

L'instrumentation présentée ici a pour but initial d'étudier les sillages de navires donc, par voie de conséquence, l'influence des bulles d'air présentes dans la mer (1), (2), (3) et (4), ainsi que la turbulence du milieu (5) (6) et (7). On rapporte ici les résultats d'expériences préliminaires réalisées dans le milieu marin en eaux abritées, sans présence de sillages, à l'aide d'un appareillage compact.

\section{2 - DISPOSITIF EXPERIMENTAL}

Il est essentiellement composé d'un flotteur cylindrique d'axe horizontal comportant à chaque extrémité sur une même génératrice et dans un même plan, deux supports de transducteurs inclinés de $45^{\circ}$ sur l'axe et faisant chacun office de quille (Figure 1). Chacun des transducteurs est formé d'une lame épaisse cylindrique demi-onde en titanate de baryum, coiffée d'une lame quart d'onde adaptatrice, le tout ayant un diamètre de $3 \mathrm{~cm}$ et résonnant sur $150 \mathrm{kHz}$. Leur diagramme de directivité dans l'eau de mer qui est donné par : $2\left(\mathrm{~J}_{1}(3 \pi \cdot \sin \phi)\right) /(3 \pi$. sin $\phi)$, est tracé figure 2 . Un générateur piloté par quartz délivre continuellement une tension sinusoïdale de $30 \mathrm{Veff}$ a $150 \mathrm{kHz}$ qui alimente l'un des transducteurs. Quant à l'autre qui travaille en récepteur, sa sortie est connectée à un amplificateur accordé sur $150 \mathrm{kHz}$ qui a une bande passante de $50 \mathrm{kHz}$ et un gain de $48 \mathrm{~dB}$. Suit alors une détection double-alternance, sans seuil, d'une constante de temps de $1 \mathrm{~ms}$. Le signal détecté est enregistré en F.M. sur bande magnétique pour être traité analogiquement en laboratoire. Comme on peut le voir sur la figure 1, le volume étudié est inférieur à vingt litres et son centre est à $50 \mathrm{~cm}$ de la surface de la mer ainsi qu'à $50 \mathrm{~cm}$ de la face active des transducteurs.

D'autre part, le signal détecté sera référencé au signal qui serait reçu si un plan parfaitement réfléchissant, parallèle à la surface, était disposé à $50 \mathrm{~cm}$ de l'axe du flotteur. Pour mesurer ce signal de référence, on immerge le flotteur horizontalement avec un lest, transducteurs vers le haut, a une profondeur de $50 \mathrm{~cm}$ dans une eau sans aucune ride et l'on connecte directement le détecteur au transducteur récepteur, sans passer par l'amplificateur. 


\section{3 - RESULTATS DES MESURES}

Les premières mesures ont été faites au mouillage le 16/06/91 à mi-flot (coeff. 84) à l'abri des vagues dans le port de Moulin-Blanc dans la rade de Brest avec un fond de $10 \mathrm{~m}$ au moment des mesures. Malgré un vent de N.O. de force 5 , les vaguelettes et les rides avaient une hauteur crête à creux inférieure à $10 \mathrm{~cm}(\mathrm{~S}=0)$ et aucun déferlement ni écume n'était apparent. Le flotteur est toujours resté immobile.

Le signal détecté $e(t)$ s'avère avoir une valeur moyenne $\bar{e}=0,615 \mathrm{~V}$, assez stable dans le temps, autour de laquelle il fluctue rapidement. Cette valeur moyenne correspond a un niveau du signal diffusé à - $42 \mathrm{~dB}$ du niveau du signal reçu après une réflexion parfaite.

On a porté sur la figure 3 le logarithme de la densité de puissance spectrale $W(F)$ de $(e(t)-\bar{e})$ en fonction linéaire de la fréquence $F$. Les points mesurés, résultats d'une moyenne du signal sur deux minutes, admettent une droite de régression qui permet de modéliser $W(F)$ par la relation $\mathrm{W}(\mathrm{F})=\mathrm{A} \cdot \exp (-\mathrm{B} . \mathrm{F})$ où $\mathrm{A}=4,5 \cdot 10^{-3} \mathrm{~V} 2 / \mathrm{Hz}$ et $\mathrm{B}=0,14 \mathrm{~s}$. Cette expression conduit à une fonction d'autocorrélation $R(T)=2 A B /\left(B^{2}+(2 \pi T)^{2}\right)$ qui est représentée sur la figure 4 où l'on a aussi porté les mesures faites à l'aide d'un intercorrélateur. Pour $T=0, R(0)=6,4.10^{-2} V^{2}$ qui est en accord avec la valeur 2A/B. Remarquons aussi que le spectre $W(F)$ conduit à une fréquence moyenne $\bar{F}=\left(\int F \cdot \exp (-B F) \cdot d F\right) /\left(\int \exp (-B F) \cdot d F\right)$ soit encore à $\bar{F}=1 / B=7,14 \mathrm{~Hz}$. La fréquence $\bar{F}$ observée en comptant par unité de temps les passages de $e(t)$ par sa valeur moyenne $\bar{e}$, est $\overline{\mathrm{F}}_{\mathrm{OBS}}=7,7 \mathrm{~Hz}$.

D'autre part, l'étude statistique du signal $\mathrm{e}(\mathrm{t})$ a été faite en mesurant sur deux minutes la fonction de distribution $P(e)$ à l'aide d'un comparateur de tension et d'un intégrateur. Cette fonction $P(e)$ est présentée sur la figure 5. Quant à sa dérivée p(e), qui est la fonction de densité de probabilité, elle est figurée en trait plein figure 6. Afin de voir si e(t) peut être un signal gaussien centré sur e, on a porté $\mathrm{P}(\mathrm{e})$ sur un graphique gausso-arithmétique reproduit figure 7. En prenant la pente moyenne à $50 \%$, on trouve un écart type $\sigma=0,26 \mathrm{~V}$. Avec cette dernière valeur, on a tracé en tirets sur la figure 6, pour comparaison, la courbe $p(e)=\left(\exp \left(-(e-\bar{e})^{2} / 2 \sigma^{2}\right)\right) / \sigma \sqrt{2 \pi}$ avec $\bar{e}=0,615 \mathrm{~V}$.

\section{4 - DISCUSSION DES RESULTATS}

En ce qui concerne le niveau moyen $\bar{e}(-42 \mathrm{~dB})$ du signal diffusé orthogonalement, il est trop intense pour n'être dû qu'à des inhomogénéités du milieu puisque celles-ci ne donnent théoriquement pas de diffusion orthogonale ((5) p. 930) et (6). Il semble qu'il faille prendre alors en considération les hétérogénéités dues aux nombreuses bulles d'air d'un diamètre de $40 \mu \mathrm{m}$ qui sont toujours présentes dans la mer et qui résonnent à $150 \mathrm{kHz}$ en diffusant l'onde isotropiquement.

Quant au spectre de puissance W(F), la modélisation exponentielle proposée rend bien compte des mesures comme le montre l'agréement avec la fonction d'autocorrélation $R(T)$ et avec la fréquence moyenne F. En traçant $\log (W)$ en fonction de $\log (F)$ sur la figure 8, l'allure parabolique est identique au spectre des vitesses de cisaillement obtenu dans la mer par N.S. OAKEY et J.A. ELLIOT (dans (8) p. 129). Comme ces auteurs, nous avons porté en tirets sur la figure 8 la courbe de NASMYTH juste décalée en ordonnées de 3,14. Ainsi les fluctuations seraient dues à la turbulence convective homogène et isotrope dans la couche de mélange de la mer et les bulles entraînées par les tourbillons, joueraient le rôle de "marqueurs".

Rien de naturel alors à ce que $e(t)$ soit gaussien comme on semble le voir sur la figure 6. Dans ce cas, $R(0)=\sigma^{2}$ et $\sigma$ devrait être égal à $0,253 \mathrm{~V}$, valeur en accord avec $\sigma=0,26 \mathrm{~V}$ trouvée par ailleurs (figure 7). Dans ce cas également, $\sigma$ peut être mesuré directement d'après A. PAPOULIS ((9) p. 481) en faisant une détection double-alternance sans seuil du signal (e(t)-e) et en moyennant avec un intégrateur sa tension de sortie $v$ qui donne $\bar{v}=\sigma \sqrt{2 / \pi}$. Avec cette méthode on a mesuré $\sigma=0,255 \mathrm{~V}$, ce qui conduit à un rapport $\sigma / \overline{\mathrm{e}} \mathrm{de}-7,6 \mathrm{~dB}$.

Enfin comme la longueur d'onde utilisée est de $1 \mathrm{~cm}$, on peut estimer la vitesse moyenne des hétérogénéités à environ $10 \mathrm{~cm} / \mathrm{s}$ à partir de $\mathrm{F}$ et en tenant compte de la géométrie du dispositif. 


\section{5 - CONCLUSION}

Pour les mesures in situ, il semble que l'on ait seulement besoin de mesurer les trois paramètres $\overline{\mathrm{e}}$, $\sigma$ et $\overline{\mathrm{F}}$. Ainsi lors des prochaines campagnes a la mer, le matériel embarqué sera plus réduit et le dépouillement des données aussi. D'autre part, il y aurait lieu de développer une théorie de la diffusion des ondes acoustiques par un milieu diphasique résonnant (bulles) et turbulent (avec et sans courant) pour mieux rendre compte des mesures.

\section{BIBLIOGRAPHIE}

(1) GILLES M.A., "Synthèse bibliographique sur les propriétés acoustiques des bulles et leur influence sur la propagation en milieu marin", 7ème Colloque GRETSI Nice 1979-pp11/1,11/6.

(2) ARZELIES P., "Propagation des ondes dans un milieu peuple de diffuseurs isotropes-nuage de bulles-", Rev.CETHEDEC n`60,1979-pp135,145.

(3) GAZANHES C., ARZELIES P., LEANDRE J., "Propagation acoustique dans un milieu diphasique eau-bulles d'air. Application à la caractérisation d'un milieu diphasique par voie acoustique", ACUSTICA vol.55,1984-pp113,122.

(4) BREITZ N., MEDWIN H., Instrumentation for in situ acoustical measurements of bubble spectra under breaking waves", J.Acoust.Soc.Am.vol.86,(2)Août 1989-pp 739,743.

(5) CLIFFORD S.F., BROWN E.H., "Acoustic scattering from a moving turbulent medium", J.Acoust.Soc.Am vol 55,n 5,Mai 1974-pp929,933.

(6) BAERG W., SCHWARTZ W.H., "Measurements of the scattering of sound from turbulence", J.Acoust.Soc.Am vol.39,n'6,1966-pp1125,1132.

(7) PELECH I., ZIPFEL G.G., HOLFORD R.L., "A wake-scattering experiment in thermally stratified water", J.Acoust.Soc.Am vol. 73,n², Fev.1983-pp 528,538.

(8) NIHOUL J.C.J., "Marine Turbulence", ELSEVIER Oceanography series 28,1980.

(9) PAPOULIS A., "Probability, random variables and stochastic processes, Mc.GRAW-HILL NewYork 1965.

\section{EIGURES}

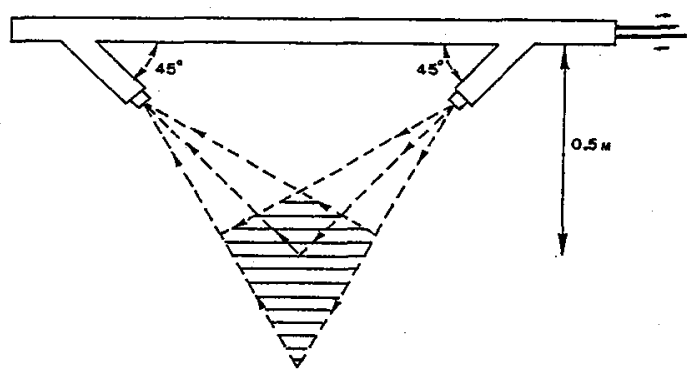

Fig. 1 : Géométrie du dispositif

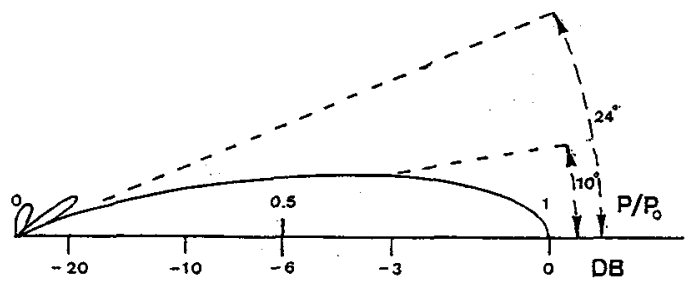

Fig. 2 : Directivité des transducteurs 


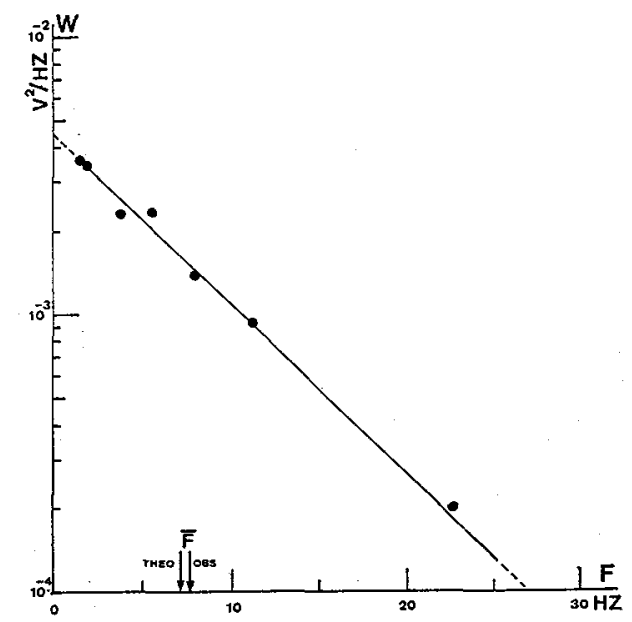

Fig. 3 : Spectre de puissance (Log-Lin)

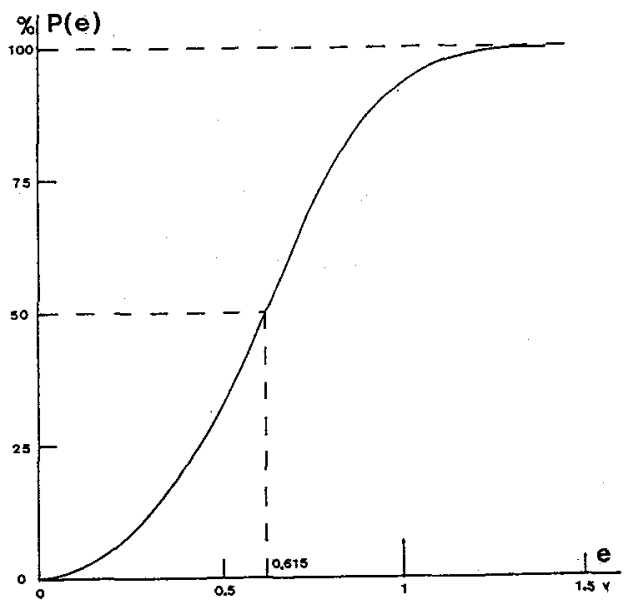

Fig. 5 : Fonction de distribution

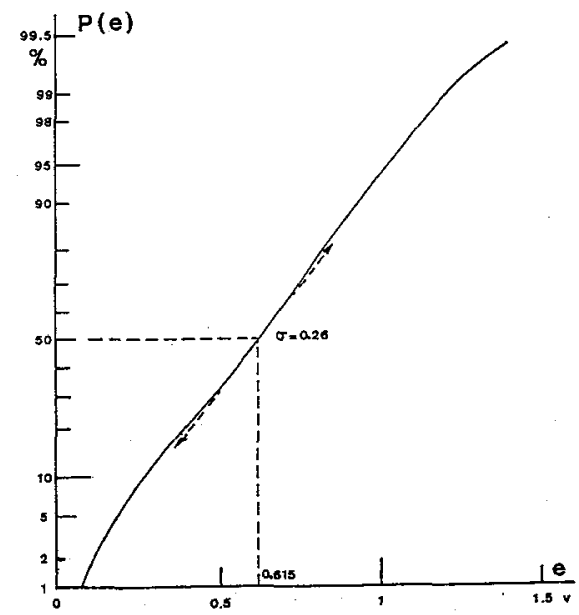

Fig. 7 : Graphe Gausso-arithmétique

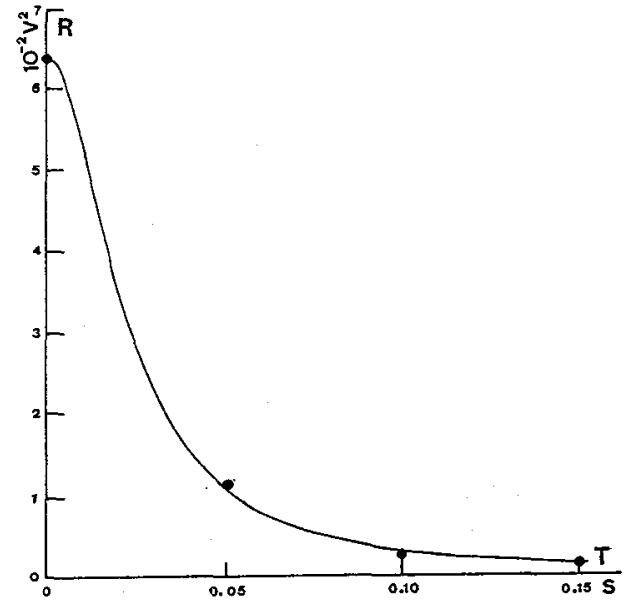

Fig. 4 : Fonction d'autocorrélation

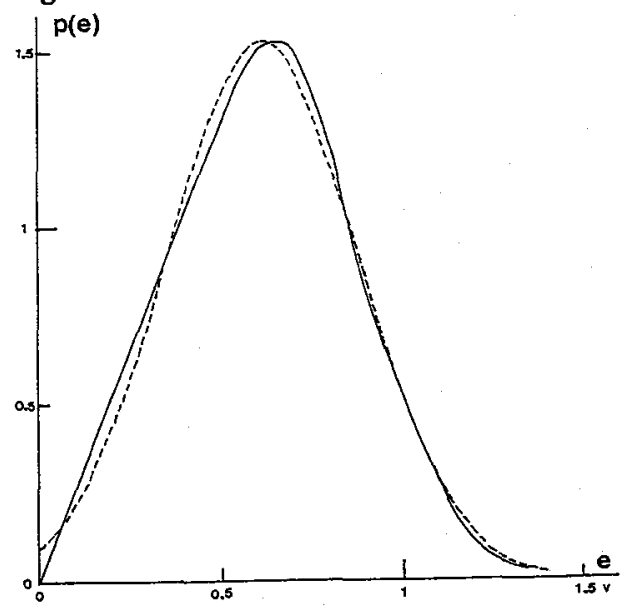

Fig. 6 : Densité de probabilité

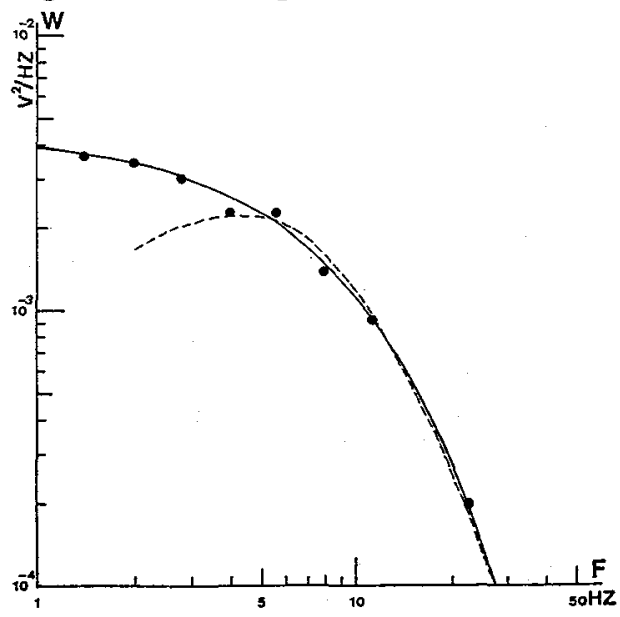

Fig. 8 : Spectre de puissance (Log-Log) 